\title{
Redox/pH dual-controlled release of chlorhexidine and silver ions from biodegradable mesoporous silica nanoparticles against oral biofilms
}

This article was published in the following Dove Press journal: International Journal of Nanomedicine

\author{
Meng-meng Lu' ${ }^{1,2}$ \\ Yuran $\mathrm{Ge}^{1,2}$ \\ Jing Qiu ${ }^{1,2}$ \\ Dan Shao 3 \\ Yue Zhang ${ }^{4}$ \\ Jing $\mathrm{Bai}^{4}$ \\ Xiao Zheng ${ }^{5}$ \\ Zhi-min Chang ${ }^{3}$ \\ Zheng Wang ${ }^{3}$ \\ Wen-fei Dong ${ }^{3}$ \\ Chun-bo Tang ${ }^{1,2}$ \\ 'Department of Oral Implantology, \\ Affiliated Hospital of Stomatology, Nanjing \\ Medical University, Nanjing, 210029 , China; \\ ${ }^{2}$ Jiangsu Key Laboratory of Oral Diseases, \\ Nanjing Medical University, Nanjing, 210029 \\ China; ${ }^{3}$ CAS Key Laboratory of Bio-Medical \\ Diagnostics, Suzhou Institute of Biomedical \\ Engineering and Technology, Chinese \\ Academy of Sciences, Suzhou, 215163 \\ China; ${ }^{4}$ School of Materials Science \\ and Engineering, Southeast University, \\ Nanjing, 211189 , China; ${ }^{5}$ Department of \\ Pharmacology, Nanomedicine Engineering \\ Laboratory of Jilin Province, College of \\ Basic Medical Sciences, Jilin University, \\ Changchun, I3002I, China
}

Correspondence: Chun-bo Tang Department of Oral Implantology, Affiliated Hospital of Stomatology, Nanjing Medical University, 136 Hanzhong Street, Nanjing Jiangsu, 210029 , China

Tel +86258503 1834

Email cbtang@njmu.edu.cn

Dan Shao

CAS Key Laboratory of Bio-Medical

Diagnostics, Suzhou Institute of Biomedical

Engineering and Technology, Chinese Academy

of Sciences, 88 Keling Road, Suzhou, Jiangsu,

215163 , China

Tel +8651269588307

Email stanauagate@outlook.com
Background: Oral plaque biofilms pose a threat to periodontal health and are challenging to eradicate. There is a growing belief that a combination of silver nanoparticles and chlorhexidine (CHX) is a promising strategy against oral biofilms.

Purpose: To overcome the side effects of this strategy and to exert maximum efficiency, we fabricated biodegradable disulfide-bridged mesoporous silica nanoparticles (MSNs) to co-deliver silver nanoparticles and CHX for biofilm inhibition.

Materials and methods: CHX-loaded, silver-decorated mesoporous silica nanoparticles (Ag-MSNs@CHX) were fabricated after CHX loading, and the pH- and glutathione-responsive release profiles of $\mathrm{CHX}$ and silver ions along with their mechanism of degradation were systematically investigated. Then, the efficacy of Ag-MSNs@CHX against Streptococcus mutans and its biofilm was comprehensively assessed by determining the minimum inhibitory concentration, minimum bactericidal concentration, minimal biofilm inhibitory concentration, and the inhibitory effect on $S$. mutans biofilm formation. In addition, the biosafety of nanocarriers was evaluated by oral epithelial cells and a mouse model.

Results: The obtained Ag-MSNs@CHX possessed redox/pH-responsive release properties of CHX and silver ions, which may be attributed to the redox-triggered matrix degradation mechanism of exposure to biofilm-mimetic microenvironments. Ag-MSNs@CHX displayed dose-dependent antibacterial activity against planktonic and clone formation of S. mutans. Importantly, Ag-MSNs@CHX had an increased and long-term ability to restrict the growth of S. mutans biofilms compared to free CHX. Moreover, Ag-MSNs@CHX showed less cytotoxicity to oral epithelial cells, whereas orally administered Ag-MSNs exhibited no obvious toxic effects in mice.

Conclusion: Our findings constitute a highly effective and safe strategy against biofilms that has a good potential as an oral biofilm therapy.

Keywords: biodegradable mesoporous silica nanoparticles, silver nanoparticles, chlorhexidine, GSH, pH-responsive release, biofilm, biocompatibility

\section{Introduction}

Periodontal diseases are common and highly prevalent chronic diseases associated with the formation and persistence of microbial biofilms that trigger an immune response, leading to periodontal tissue destruction, alveolar bone resorption, and eventual tooth loss. ${ }^{1-3}$ Biofilms are characterized as a self-produced protective sheet of extracellular matrix consisting of robust bacterial cells, providing impermeability against biological, chemical, and mechanical stress. ${ }^{4,5}$ A triad of oral anaerobic bacteria, including Porphyromonas gingivalis, Streptococcus mutans, and Fusobacterium nucleatum, was identified as a 
well-established player in the development of oral microbial biofilms. ${ }^{6,7}$ Among them, S. mutans is the predominant etiological pathogen that is firmly adherent to tooth surfaces and enmeshed in a three-dimensional matrix of exopolysaccharides to develop biofilms. ${ }^{8,9}$ Simultaneously, these embedded pathogens play a critical role in producing highly acidic niches with $\mathrm{pH}$ values close to 4.5 , resulting in an acidic microenvironment that ensures continuous biofilm accumulation. ${ }^{10}$ Current chemical-mediated approaches against oral biofilms are restricted to conventional antiseptics that are less efficient for degrading the extracellular matrix. ${ }^{11,12}$ Moreover, metal nanomaterials have been considered as promising strategies to inhibit biofilm formation by reducing bacterial adhesion and viability. ${ }^{13,14}$ Therefore, there is a growing belief that a combination of antibacterial agents is a promising strategy against oral infections, which may increase biofilm inhibition and reduce the ever-increasing risk of antibacterial resistance. Among several antibacterial agents, chlorhexidine (CHX) is considered the "gold standard" of oral antiseptics with broadspectrum efficacy; however, CHX is not recommended for long-term usage because of sustained administration-induced side effects, including taste disturbances and staining of the teeth and mucosa. ${ }^{15-17}$ The obvious antibacterial effect of silver nanoparticles (Ag NPs) has been known for several years, and it can lead to the loss of bacterial cell membrane integrity and cell wall permeability, particularly the blocking of bacterial adhesion and biofilm formation. ${ }^{13,18-20}$ Importantly, the combination of CHX and Ag NPs has a synergistic antibacterial effect and improved biofilm inhibitory activity. ${ }^{21,22}$ However, side effects occurring from the long-term coadministration without targeting the biofilm microenvironment may limit its further clinical applications. ${ }^{23}$ In this regard, a promising drug co-delivery system is highly desirable for the controlled release of $\mathrm{CHX}$ and silver ions in response to a pathological environment in the oral cavity. This co-delivery system may show maximum synergistic efficiency to overcome biofilm recalcitrance and ameliorate the side effects.

Because of the integration of nanotechnology in biomedicine, several nanocarriers were intelligently designed for the stimuli-responsive controllable release of multiple therapeutic agents at the targeted site while simultaneously reducing the side effects. ${ }^{24-27}$ Mesoporous silica nanoparticles (MSNs) have gained substantial attention as promising inorganic nanocarriers for controlled drug delivery because of their large surface area, tunable pore size, and easy surface functionalization. ${ }^{28-35}$ In particular, MSNs show the $\mathrm{pH}$-activated release of antibacterial agents to achieve efficient bacterial killing. ${ }^{36-40}$ However, the slow degradation of MSNs in biological systems has not yet been fully addressed and remains a major obstacle impeding their further clinical translation. ${ }^{41}$ Recently, our group have introduced disulfide bond-bridged organosilica moieties into the silica framework to fabricate biodegradable MSNs, which exhibit $\mathrm{pH}$-responsive drug release for efficient and safe cancer therapy. ${ }^{42}$ Importantly, these organo-bridged MSNs with precisely tuned degradable properties also exhibit matrix degradation controlled drug release through glutathione (GSH)-induced disulfide bond cleavage. ${ }^{43-45}$ Therefore, it is of profound importance that $\mathrm{GSH} / \mathrm{pH}$ dual responsiveness and controlled release can be achieved simultaneously in these biodegradable MSNs. Our laboratories collectively explored biocompatible nanosilver-decorated MSNs using a facile and environmentally friendly method. ${ }^{46}$ These nanocarriers could load both $\mathrm{CHX}$ and silver with a $\mathrm{pH}$-triggered drug release, leading to synergistic antibacterial effects against both Gram-positive Staphylococcus aureus and Gram-negative Escherichia coli. However, the therapeutic effects of our nanoantiseptics on biofilms have not yet been explored.

Considering this, the goal of the present study was to investigate whether these biodegradable nanoantiseptics could achieve the redox/pH dual-controlled release of $\mathrm{CHX}$ and silver ions against $S$. mutans biofilms (Figure 1). In this proof-of-concept study, we present silver-decorated MSNs (Ag-MSNs) for CHX loading and systematically investigate the $\mathrm{pH}$ - and GSH-responsive release profiles of CHX and silver ions along with their mechanism of degradation. Then, the efficacy of CHX-loaded, silver-decorated mesoporous silica nanoparticles (Ag-MSNs@CHX) against S. mutans and its biofilm is comprehensively assessed by determining the minimum inhibitory concentration (MIC), minimum bactericidal concentration (MBC), minimal biofilm inhibitory concentration (MBIC), and the inhibitory effect on S. mutans biofilm formation. In addition, the biosafety of nanocarriers is evaluated by oral epithelial cells and a mouse model.

\section{Materials and methods Materials}

Tetraethyl orthosilicate (TEOS, 98\%), triethanolamine, cetyltrimethylammonium tosylate (98\%), 3-aminopropyltriethoxysilane, and CHX (99.5\%) were purchased from SigmaAldrich Co. (St Louis, MO, USA). Silver nitrate $\left(\mathrm{AgNO}_{3}\right.$, $99.5 \%)$, dimethyl sulfoxide, hydrochloric acid $(37 \%)$, $N, N$-dimethylformamide, anhydrous ethanol (EtOH), and ammonium hydroxide $\left(\mathrm{NH}_{4} \mathrm{OH}, 28 \%\right)$ were purchased from 


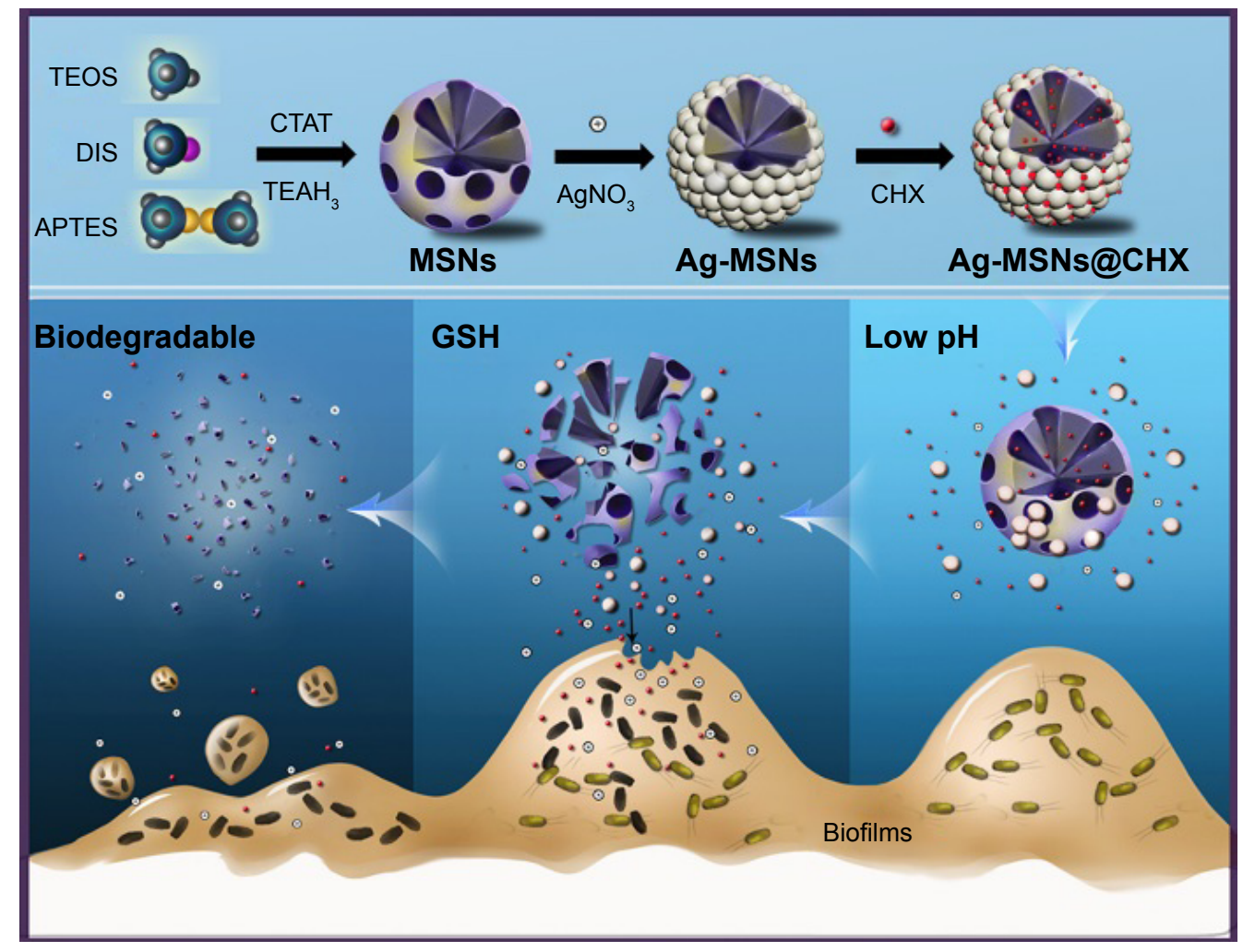

Figure I Schematic representation of synthetic diagram for fabrication of Ag-MSNs@CHX and their redox/pH-responsive release properties of CHX and silver ions against oral biofilms.

Abbreviations: Ag-MSNs, silver-decorated mesoporous silica nanoparticles; Ag-MSNs@CHX, chlorhexidine-loaded, silver-decorated mesoporous silica nanoparticles; APTES, 3-aminopropyltriethoxysilane; CHX, chlorhexidine; CTAT, cetyltrimethylammonium tosylate; DIS, bis(3-triethoxysilyl propyl)disulfide; GSH, glutathione; MSNs, mesoporous silica nanoparticles; $\mathrm{TEAH}_{3}$, triethanolamine; $\mathrm{TEOS}$, tetraethyl orthosilicate.

Beijing Chemical Reagent Co. (Beijing, China). Promega ${ }^{\mathrm{TM}}$ CellTiter 96 ${ }^{\mathrm{TM}}$ AQueous One Solution Cell Proliferation Assay (MTS) kits were purchased from Thermo Fisher Scientific (Waltham, MA, USA). DMEM, Roswell Park Memorial Institute-1640 medium, FBS, penicillin, and streptomycin were obtained from Thermo Fisher Scientific.

\section{Synthesis and biodegradable characterization of Ag-MSNs}

The disulfide-bridged MSNs were synthesized using a wellknown modification of the sol-gel method, which is based on the incorporation of a disulfide silica precursor into the framework of MSNs. ${ }^{46}$ Briefly, $0.75 \mathrm{~g}$ of cetyltrimethylammonium tosylate was first mixed with $0.25 \mathrm{~g}$ of triethanolamine in $50 \mathrm{~mL}$ of deionized water, and the mixture was stirred at $80^{\circ} \mathrm{C}$ for 1 hour. When the surfactant was completely dissolved, a mixture of $0.5 \mathrm{~g}$ of TEOS, $0.5 \mathrm{~g}$ of bis(3-triethoxysilyl propyl)disulfide, and $0.1 \mathrm{~g}$ of 3 -aminopropyltriethoxysilane was quickly added into the surfactant solution and reacted for another 3 hours at the same temperature. After purification, nanosilver-decorated MSNs were prepared using a facile ultrasonication-assisted method according to our previous study. ${ }^{46}$ Transmission electron microscopy (TEM) images were obtained with a JEM-2100F transmission electron microscope (JEOL, Tokyo, Japan) under a $200 \mathrm{kV}$ accelerating voltage. The morphologies of the Ag-MSNs and Ag-MSNs with bacteria were observed by using a scanning electron microscope (SEM; field emission SEM, S4800; Hitachi Ltd., Tokyo, Japan). The Ag concentration was measured with an inductively coupled plasma mass spectrometer (Xseries II; Thermo Fisher Scientific). The degradation of MSNs and Ag-MSNs was determined by mimicking intracellular GSH concentrations $(5 \mathrm{mM})$ in deionized water at $37^{\circ} \mathrm{C}$ under constant rotation. After incubation for 0,1 , and 4 days, each sample was collected and observed by TEM.

\section{$\mathrm{CHX}$ loading and the release profile of $\mathrm{CHX}$ and silver ions}

Carboxylate group-functionalized Ag-MSNs were prepared to load $\mathrm{CHX}$ and achieve GSH/pH dual-sensitive $\mathrm{CHX}$ 
release according to our previous work. ${ }^{29}$ To achieve high CHX loading, the CHX solution $(2.5 \mathrm{mg} / \mathrm{mL})$ was freshly prepared by dissolving $12.5 \mathrm{mg}$ of CHX in $5 \mathrm{~mL}$ of ethanol. Then, $5 \mathrm{~mL}$ of Ag-MSNs-COOH ethanol solution $(5 \mathrm{mg} / \mathrm{mL})$ was added to the CHX solution and the mixture was stirred at room temperature for 24 hours. CHX-loaded Ag-MSNs were washed with deionized water, and the amount of $\mathrm{CHX}$ was measured by ultraviolet-visible spectrophotometry at a wavelength of $254 \mathrm{~nm}$. The loading efficiency and the drug-loading content were calculated according to our previous studies. ${ }^{29,46}$

To investigate the release behavior of $\mathrm{CHX}$ and silver ions,Ag-MSNs@CHX (100 $\mu \mathrm{g} / \mathrm{mL})$ were dispersed in simulated body fluid (SBF) solutions ( $\mathrm{pH}=7.4$ or 5.5$)$ with or without $5 \mathrm{mM}$ GSH. Then, the mixture was transferred into a dialysis bag (molecular weight cutoff 5,000) on a shaking table at $37^{\circ} \mathrm{C}$ for 4 days. At each time interval $(0,1,2,3,4,5$, $6,12,24,48,72$, and 96 hours), the amount of released $\mathrm{CHX}$ was determined by ultraviolet-visible spectrophotometry. The amount of released silver ions at $0,6,12,24,48,72$, and 96 hours was measured by inductively coupled plasma mass spectrometer.

\section{Antibacterial properties}

Standard strains of $S$. mutans ATCC 25175 were obtained from the archival collection at the Jiangsu Key Laboratory of Oral Diseases, Nanjing Medical University. S. mutans was cultured on brain heart infusion (BHI)-S blood agar plates (BHI, Oxoid; supplemented with $5 \mathrm{mg} / \mathrm{mL}$ yeast extract, $5 \mathrm{mg} / \mathrm{mL}$ hemin, and $0.2 \mathrm{mg} / \mathrm{mL}$ menadione) under standard anaerobic conditions $\left(80 \% \mathrm{~N}_{2}, 10 \% \mathrm{H}_{2}\right.$, and $10 \% \mathrm{CO}_{2}$ at $37^{\circ} \mathrm{C}$ ). A single bacterial colony was inoculated into $10 \mathrm{~mL}$ of BHI broth and was cultured at $37^{\circ} \mathrm{C}$ for 24 hours under a $5 \% \mathrm{CO}_{2}$ atmosphere.

To measure the MIC, Ag-MSNs@CHX at different concentrations $(50,25,12.5,6.25,3.125$, and $0 \mu \mathrm{g} / \mathrm{mL})$ and $50 \mu \mathrm{g} / \mathrm{mL}$ of MSNs were separately added into 24-well plates with $1 \mathrm{~mL}$ of $S$. mutans culture solution $\left(1 \times 10^{5}\right.$ colony-forming units [CFU]/mL) and shaken under appropriate conditions. At different time intervals, $100 \mu \mathrm{L}$ of the medium was collected and transferred to a 96-well plate to detect the OD value at $600 \mathrm{~nm}$. To measure the MBC, bacterial colonies were formed by plating the treated samples of microbial cells on BHI-agar growth plates. In brief, 50, $25,12.5,6.25,3.125$, or $0 \mu \mathrm{g} / \mathrm{mL}$ of Ag-MSNs@CHX were each mixed with $1 \times 10^{5} \mathrm{CFU} / \mathrm{mL}$ bacteria in BHI medium. Then, a total of $200 \mu \mathrm{L}$ of bacteria-NP medium was plated on a single $\mathrm{BHI}$-agar growth plate and incubated overnight at $37^{\circ} \mathrm{C}$. Colonies were observed after 24 hours, and digital images of each plate were captured.

\section{Anti-biofilm assay}

The 1-day-old biofilms were prepared in chambered cover glass according to a previous protocol. A volume of $200 \mu \mathrm{L}$ of $S$. mutans $\left(1 \times 10^{8} \mathrm{CFU} / \mathrm{mL}\right)$ suspension was seeded in 96-well plates and incubated in an anaerobic chamber at $37^{\circ} \mathrm{C}$. Ag-MSNs or Ag-MSNs at different concentrations (100, 50, $25,12.5,6.25$, and $3.125 \mu \mathrm{g} / \mathrm{mL}$ ) and $\mathrm{CHX}$ dosed assuming a $20 \%$ loading content $(20,10,5,2.5,1.25,0.625 \mu \mathrm{g} / \mathrm{mL})$ were added to each well. After 24 hours of incubation, the medium was slowly aspirated without disturbing the biofilm to remove the planktonic bacteria. Then, the viability of cells in the biofilm was determined by MTS assay, and the untreated cells were used as controls. The OD of each well was measured at $490 \mathrm{~nm}$, and the cell viability ratio of the control was calculated using the following formula: $\mathrm{A} / \mathrm{B} \times 100 \%$, where $\mathrm{A}$ is the $\mathrm{OD}$ value from the experimental groups and $\mathrm{B}$ is the OD value from the control groups. To investigate the long-term anti-biofilm effect, Ag-MSNs@CHX $(50 \mu \mathrm{g} / \mathrm{mL})$, $\mathrm{AgNO}_{3}(2.65 \mu \mathrm{g} / \mathrm{mL}), \mathrm{Ag}-\mathrm{MSNs}(50 \mu \mathrm{g} / \mathrm{mL})$, and CHX (10 $\mu \mathrm{g} / \mathrm{mL})$ were added to the media, and the cell viability was measured after 24, 48, or 72 hours of incubation.

Bacterial biofilm formation was determined by confocal laser scanning microscopy and SEM. S. mutans was cultivated and left to grow for 24 hours in BHI supplemented with $1 \%$ sucrose in a 12 -well microtiter plate (Nunc, Rochester, NY, USA). After treating with Ag-MSNs@CHX $(50 \mu \mathrm{g} / \mathrm{mL}), \mathrm{AgNO}_{3}(2.65 \mu \mathrm{g} / \mathrm{mL}), \operatorname{Ag}-\mathrm{MSNs}(50 \mu \mathrm{g} / \mathrm{mL})$, or CHX $(10 \mu \mathrm{g} / \mathrm{mL})$ for 24 hours, the samples were stained with the LIVE/DEAD Bacterial Viability Kit (Thermo Fisher Scientific) and examined by confocal laser scanning microscopy (Olympus FV10-ASW; Olympus Corporation, Tokyo, Japan). To observe the morphology of the biofilms, the formed biofilms were lightly rinsed three times with PBS and fixed with glutaraldehyde $(2.5 \% \mathrm{v} / \mathrm{v})$ at $4^{\circ} \mathrm{C}$. After 4 hours of incubation, the samples were washed in PBS and then dehydrated in a series of ethanol solutions $(50 \%, 70 \%$, $95 \%$, and $100 \%$ ) for 10 minutes each. The samples were then sputter-coated with gold and observed using SEM.

\section{Cytotoxicity assessment}

Human immortalized oral epithelial cells (HIOECs) were a kind gift from Prof Wantao Chen (Shanghai Jiaotong University), which were developed and characterized in a 
previous work. ${ }^{47}$ The Ethics Committee of Affiliated Hospital of Stomatology of Nanjing Medical University approved the use of HIOECs in our study. HIOECs were cultured in DMEM supplemented with $10 \%(\mathrm{v} / \mathrm{v})$ heat-inactivated FBS, penicillin $(100 \mathrm{U} / \mathrm{mL})$, and streptomycin $(100 \mu \mathrm{g} / \mathrm{mL})$ at $37^{\circ} \mathrm{C}$ under $5 \% \mathrm{CO}_{2}$ conditions. For the cytotoxicity assay, the cells in logarithmic growth phase were seeded in a 96-well plate at a density of 5,000 cells per well overnight. Then, the cells were treated with different concentrations (100, 50, 25, 12.5, 6.25, and 3.125 $\mu \mathrm{g} / \mathrm{mL}$ ) of Ag-MSNs@CHX or Ag-MSNs, whereas the free drugs were dosed assuming $20 \%$ CHX loading $(20,10,5,2.5,1.25,0.625 \mu \mathrm{g} / \mathrm{mL})$. After 6 hours of incubation, the cell viability was assessed using the above-mentioned MTS assays.

\section{Animals and biosafety evaluation}

All animal experimental protocols were approved by the ethics committee for the Use of Experimental Animals of Jilin University. The experimental manipulation of mice was conducted in accordance with the National Institute of Health Guide for the Care and Use of Laboratory Animals and the approval of the Scientific Investigation Board of Science and Technology of Jilin Province (Changchun, China). Both sexes of ICR mice (aged 6-8 weeks) were used for the singledose exposure studies. Mice were orally administered MSNs or Ag-MSNs at $50 \mathrm{mg} / \mathrm{kg}$ dose for 30 days ( $\mathrm{n}=6$ ). The body weights were recorded once in every 3 days. All mice were sacrificed at the end of the study, and blood drawn from the eyeball was collected for blood tests. The blood cells were examined with a hemocyte counter (Coulter STKS; Coulter Electronics Corp., Hialeah, FL, USA) for white blood cells, red blood cells, hematocrit, mean corpuscular volume, hemoglobin, platelets, mean corpuscular hemoglobin, and mean corpuscular hemoglobin concentration measurements. The serum was collected for detecting the levels of aspartate aminotransferase (AST), alanine aminotransferase (ALT), alkaline phosphatase (ALP), albumin (ALB), blood urea nitrogen, serum creatinine, total cholesterol, and total triglycerides. The main organs, including the liver, spleen, kidney, heart, and lung, were collected, fixed, and stained with $\mathrm{H} \& \mathrm{E}$ for histopathologic examinations.

\section{Statistical analysis}

The experimental results were analyzed with SPSS19.0 statistical software. Representative data were expressed as $x \pm s$. The comparison among groups was conducted by using the least significant difference method, with single-factor
ANOVA (one-way ANOVA). $P<0.05$ was considered significantly different. All experiments were repeated at least three times.

\section{Results and discussion}

By co-condensating TEOS and an organo-silica precursor on a molecular level, the obtained disulfide-bridged MSNs demonstrated a uniform spherical morphology with an average diameter of $\sim 100 \mathrm{~nm}$ (Figure 2A). Ag-MSNs were subsequently fabricated by self-reducing silver nanodots on the surface of MSNs using a facile method according to our previous study. As shown in Figure 2B, silver nanodots of 2-5 $\mathrm{nm}$ in size were homogeneously distributed on the surfaces of the matrix or inside the pores. Since the incorporation of redox-active disulfide bonded into the framework of MSNs was shown to enhance their biodegradation behavior, we predicted that the disulfide-bridged framework might accelerate the release of Ag NPs in response to reduced physiological stress. ${ }^{45}$ To mimic the accumulated reducing microenvironment in S. mutans, we immersed MSNs and Ag-MSNs into a simulated $5 \mathrm{mM} \mathrm{GSH}$ solution for prolonged durations, and changes in the morphology of the samples were directly observed by TEM. As shown in Figure $2 \mathrm{C}-\mathrm{H}$, the morphology of MSNs began to change from regular to fragmented only after 24 hours of exposure, and the matrix was completely disintegrated into collapsed nanostructures after 4 days of culturing, which was induced by the cleavage of disulfide bonds in the presence of GSH. Accordingly, Ag-MSNs exhibited a similar time-dependent biodegradable behavior. Moreover, the dissociated small silver dots arising from the degraded matrix were clearly observed, which facilitated the release of silver ions and further improved the antibacterial effects. Collectively, the gradual degradation of Ag-MSNs may be promising for the controlled release of $\mathrm{CHX}$ and silver ions in a reducing microenvironment.

Our group has previously demonstrated that Ag-MSNs could load and release CHX due to their large pore size. ${ }^{46}$ In this regard, we increased the concentration of CHX to obtain Ag-MSNs@CHX with a higher drug loading content $(21.5 \% \pm 2.2 \%)$. Since the oral biofilm was characterized by locally acidic and reducing microenvironments, we expected that the stimuli triggered both the CHX and silver ion release behavior under acidic and reducing conditions. Here, the CHX and silver ion release assay were simultaneously performed in acidic $\mathrm{SBF}$ solution $(\mathrm{pH}=5.5)$ and neutral SBF solution ( $\mathrm{pH}=7.4)$ in the presence and absence of GSH $(5 \mathrm{mM})$, respectively. As shown in Figure 3A, the results 

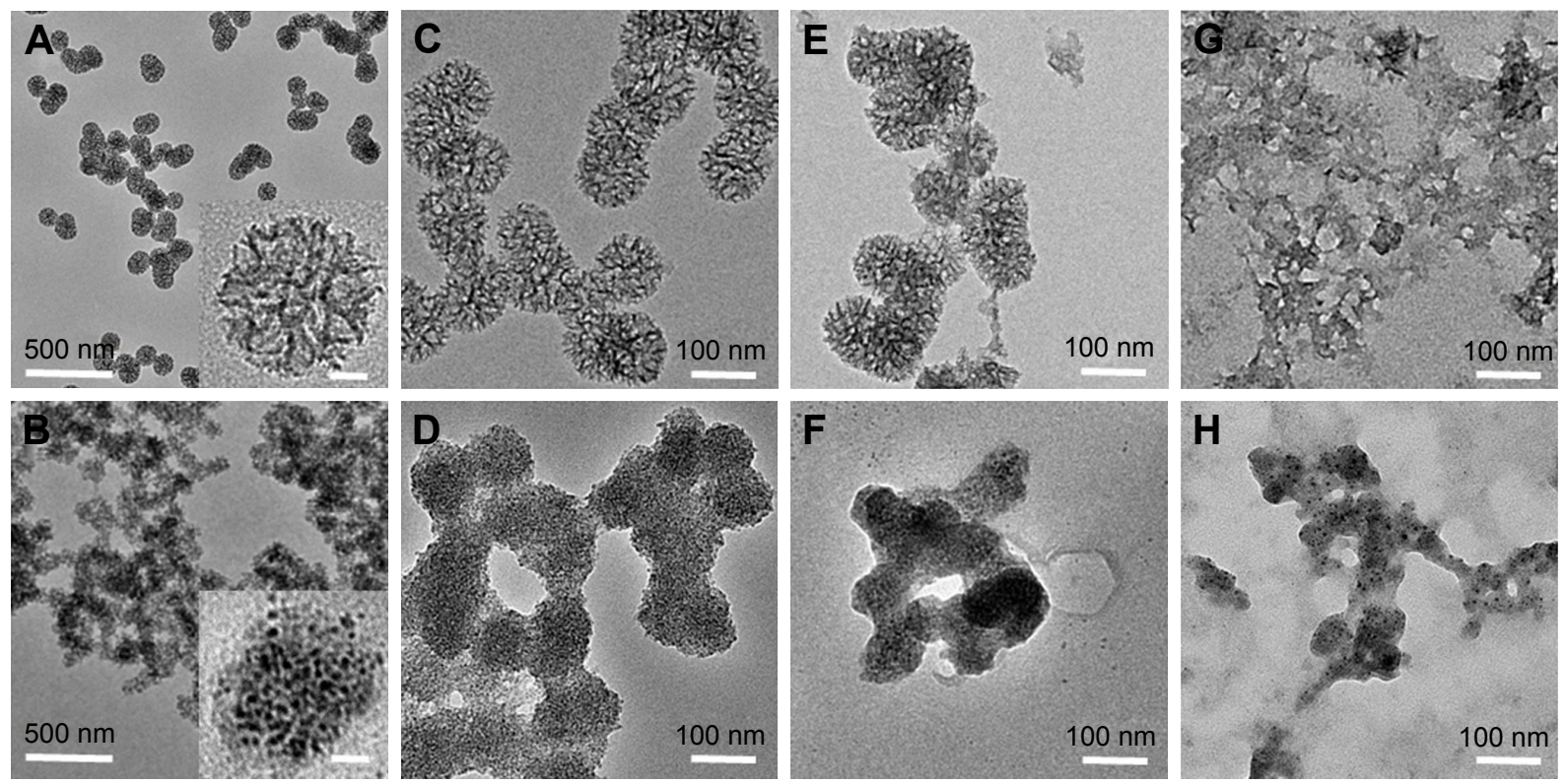

Figure 2 Biodegradation of Ag-MSNs.

Notes: TEM images of MSNs (A, C, E, and $\mathbf{G}$ ) and Ag-MSNs (B, D, F, and $\mathbf{H})$ after degradation for 0 days (C and $\mathbf{D})$, I day (E and F), and 5 days (G and $\mathbf{H})$ in GSH concentrations of $5 \mathrm{mM}$. The scale bars in the inner images of $\mathbf{A}$ and $\mathbf{B}$ represent $10 \mathrm{~nm}$.

Abbreviations: Ag-MSNs, silver-decorated mesoporous silica nanoparticles; GSH, glutathione; MSNs, mesoporous silica nanoparticles; TEM, transmission electron microscopy.

confirm our expectation that $\mathrm{CHX}$ is released in a GSH- and pH-responsive manner from Ag-MSNs@CHX. On the one hand, the $\mathrm{CHX}$ release amount became markedly accelerated after 24 hours of incubation in acid conditions, indicating the preferential release of $\mathrm{CHX}$ through the protonation and dissociation of carboxyl functional groups in acidic environments. On the other hand, the $\mathrm{CHX}$ release percentage significantly increased from 24 to 96 hours in the presence of GSH, consistent with the TEM images of degradation. In particular, the highest release of $\mathrm{CHX}$ was observed in acidic SBF in the presence of GSH, indicating the combined $\mathrm{CHX}$ release in response to the dual redox/pH environments. However, Ag-MSNs@CHX in pure SBF solution showed a slow and sustained $\mathrm{CHX}$ release percentage as low as $25 \%$.
A

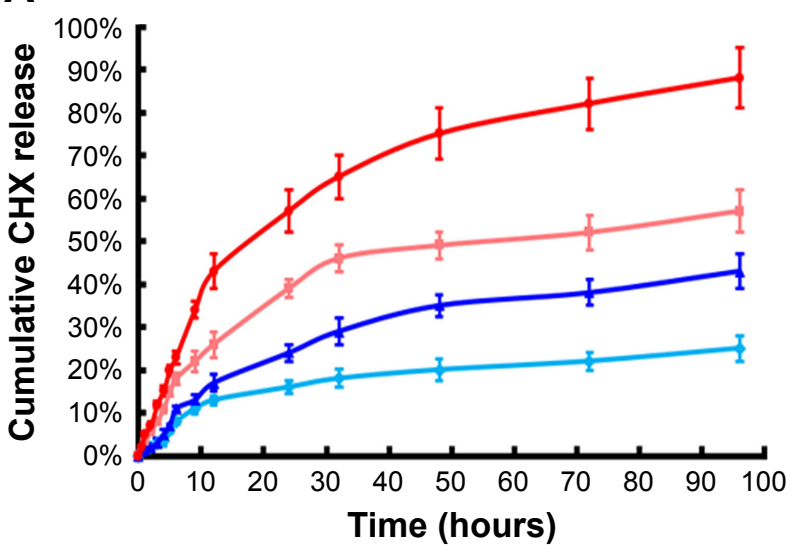

B

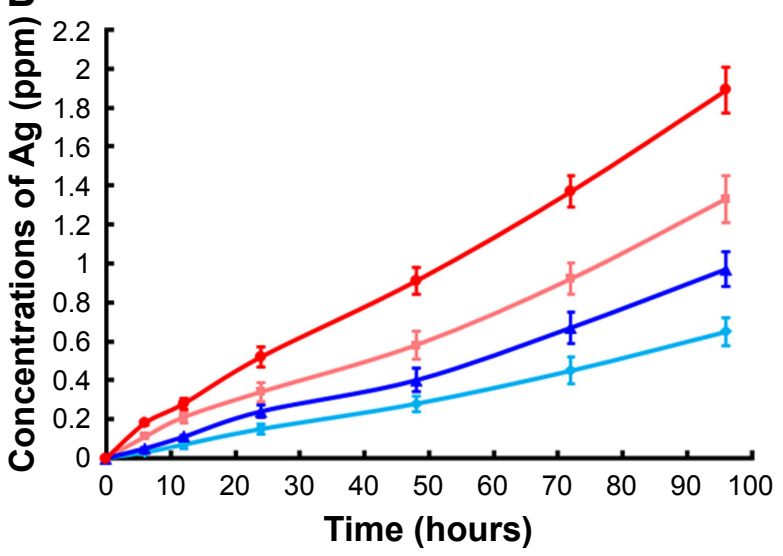

$\multimap \mathrm{pH}=7.4, \mathrm{GSH}=0 \mathrm{mM} \multimap \mathrm{pH}=5.5, \mathrm{GSH}=0 \mathrm{mM}$
$\leftarrow \mathrm{pH}=7.4, \mathrm{GSH}=5 \mathrm{mM} \multimap \mathrm{pH}=5.5, \mathrm{GSH}=5 \mathrm{mM}$

Figure 3 Release profiles of Ag-MSNs@CHX.

Note: The redox/pH-dependent release profiles of $(\mathbf{A}) \mathrm{CHX}$ and $(\mathbf{B})$ silver ions from Ag-MSNs@CHX at pH=7.0 or $5.5 \mathrm{SBF}$ solution in the absence or presence of 5 mM GSH. Abbreviations: Ag-MSNs@CHX, chlorhexidine-loaded, silver-decorated mesoporous silica nanoparticles; $\mathrm{CHX}$, chlorhexidine; GSH, glutathione; SBF, simulated body fluid. 
We continued to investigate the release profiles of silver ions fromAg-MSNs@CHX in the same stimulated conditions. As shown in Figure 3B, Ag-MSNs@CHX also showed a GSH- and pH-dependent release behavior of silver ions over a course of 4 days. Importantly, more silver ions were released into an acidic/reducing environment compared to that in acidic or reducing conditions, which might be attributed to the $\mathrm{pH}$-dependent dissociation of nanosilvers and the matrix degradation-mediated nanosilver release in response to GSH. Taken together, these findings suggest that Ag-MSNs@CHX could possess GSH-/pH-activated release of CHX and silver ions, which may enhance their inhibitory effect against $S$. mutans and its biofilms.

Since $S$. mutans was the main candidate to form oral biofilms and acid environments, we used it as a model to investigate the antibacterial activity of Ag-MSNs@CHX. We first investigated the MIC by determining the dynamic growth kinetics of S. mutans in SBI media after co-culturing with Ag-MSNs@CHX from 1 to 24 hours. As expected, the initial growth of $S$. mutans was time- and dose-dependently delayed with Ag-MSNs@CHX, while MSNs only had no effect on the growth of S. mutans (Figure 4A).Ag-MSNs@, CHX completely inhibited bacterial growth when their concentration was higher than $12.5 \mu \mathrm{g} / \mathrm{mL}$, demonstrating that the corresponding MIC value of Ag-MSNs@CHX was $12.5 \mu \mathrm{g} / \mathrm{mL}$. In addition, the dose-dependent inhibition of bacterial colonies on BHI-agar media by Ag-MSNs@CHX indicated that the corresponding MBC value of Ag-MSNs@, CHX was $25 \mu \mathrm{g} / \mathrm{mL}$ (Figure 4B). Based on these findings, we investigated the preventive activities of Ag-MSNs@ CHX against $S$. mutans biofilms using an MTS assay. As shown in Figure 4C, Ag-MSNs, CHX, and Ag-MSNs@ CHX dose-dependently inhibited the growth of $S$. mutans biofilms and the MBIC of Ag-MSNs@CHX was $50 \mu \mathrm{g} / \mathrm{mL}$. As expected, Ag-MSNs@CHX exhibited greater biofilm inhibition than Ag-MSNs or CHX at 24 hours. To further investigate the long-term antibacterial effect of Ag-MSNs@, CHX, we selected their MBIC as the standard dose and
A

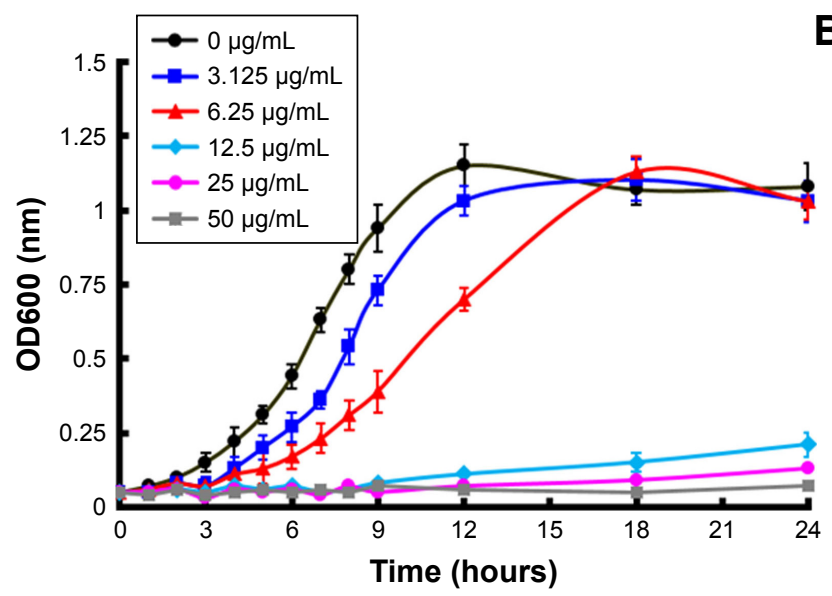

B

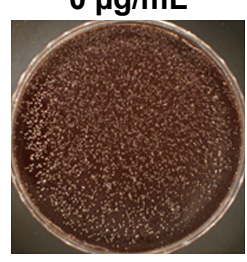

$12.5 \mu \mathrm{g} / \mathrm{mL}$

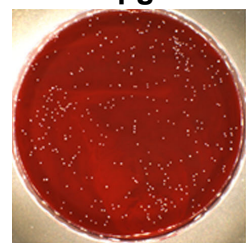

$3.125 \mu \mathrm{g} / \mathrm{mL}$

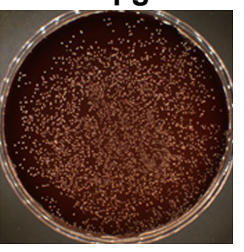

$25 \mu \mathrm{g} / \mathrm{mL}$

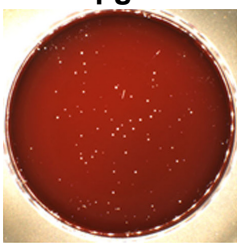

$6.25 \mu \mathrm{g} / \mathrm{mL}$

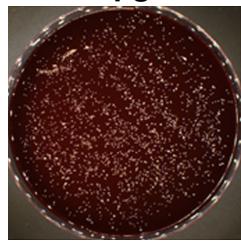

$50 \mu \mathrm{g} / \mathrm{mL}$

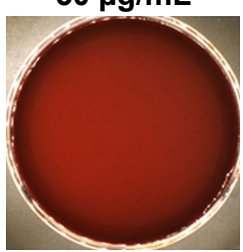

C

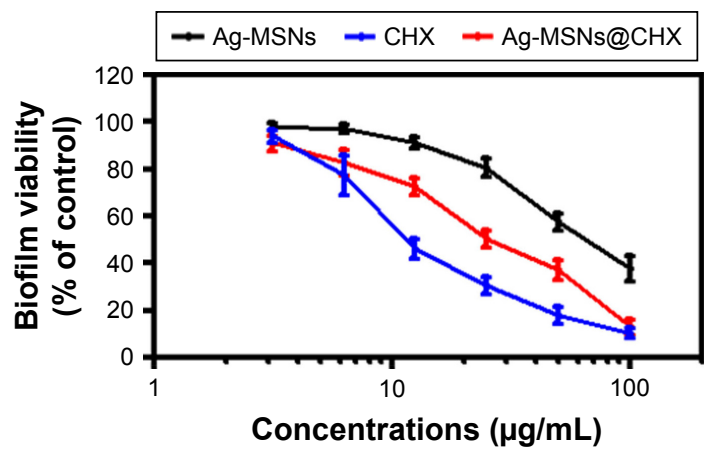

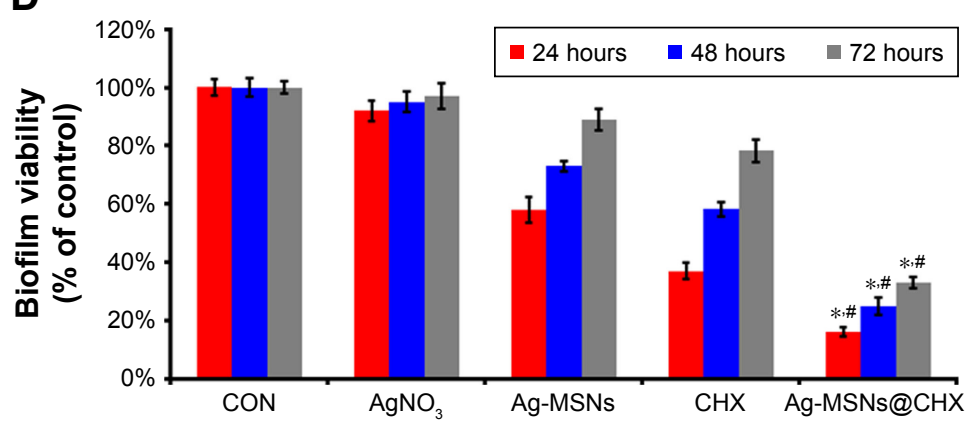

Figure 4 Antibacterial effects of Ag-MSNs@CHX.

Notes: (A) Bacterial growth curve of Streptococcus mutans in BHI liquid medium in the presence of Ag-MSNs@CHX at different concentrations. (B) Photographs of BHIagar plates coated with S. mutans supplemented with different concentrations of Ag-MSNs@CHX. (C) Anti-biofilm activity of Ag-MSNs@CHX at different concentrations against S. mutans at 24 hours. (D) Preventive activity of Ag-MSNs@CHX, Ag-MSNs, free $\mathrm{CHX}$, and $\mathrm{AgNO}_{3}$ against S. mutans biofilms. These data represent three separate experiments and are presented as mean \pm SD. $* P<0.05$ vs the control group, ${ }^{\#} P<0.05$ vs the $C H X$ group.

Abbreviations: Ag-MSNs, silver-decorated mesoporous silica nanoparticles; Ag-MSNs@CHX, chlorhexidine-loaded, silver-decorated mesoporous silica nanoparticles; $\mathrm{BHI}$, brain heart infusion; $\mathrm{CHX}$, chlorhexidine; $\mathrm{CON}$, control. 
compared their anti-biofilm activity with those of free $\mathrm{AgNO}_{3}, \mathrm{Ag}-\mathrm{MSN}$, and $\mathrm{CHX}$ with equal amounts of silver or CHX at 24, 48, and 72 hours. As shown in Figure 4D, biofilm viability decreased in all test groups, whereas the Ag-MSNs@CHX group exhibited the strongest biofilm inhibition compared to the other groups. Although the antibiofilm effect decreased with increasing incubation time, Ag-MSNs@CHX showed the best performance, even at 72 hours, which prolonged the anti-biofilm activity of $\mathrm{CHX}$. This long-term anti-biofilm property of Ag-MSNs@CHX may be due to several reasons. First, the positively charged Ag-MSNs@CHX could preferably target the negatively charged biofilm and consequently lead to $\mathrm{pH}$-sensitive $\mathrm{CHX}$ release during the first 48 hours. Second, the GSH-triggered matrix degradation could facilitate the release of Ag NPs and the dissolution of silver ions primarily after 48 hours. These findings indicate that the sequential release of $\mathrm{CHX}$ and silver ions may exert maximum synergistic anti-biofilm efficiency, particularly in the long term. We further used LIVE/DEAD staining and SEM assay to study the manner in which Ag-MSNs@CHX influence S. mutans biofilm formation. In the LIVE/DEAD staining method, live bacteria with intact cell membranes are stained green, whereas dead bacteria with damaged membranes are stained red. As shown in Figure 5, the strongest red fluorescence and weakest green fluorescence of the $S$. mutans biofilm were observed in the presence of Ag-MSNs@CHX, indicating that the amount of dead or compromised bacteria was increased, consistent with the results of a standard viability assay. The SEM assay clearly showed the morphology and structure of the $S$. mutans biofilm (Figure 6). The biofilm without any treatment exhibited grape-like colonies, whereas Ag-MSNs@CHX-treated biofilms presented obvious structural destruction, and most of the bacteria were killed, suggesting that biofilm formation was indeed inhibited. As observed in Figures 5 and 6,Ag-MSNs@CHX exhibited higher anti-biofilm properties than Ag-MSNs or CHX. This phenomenon might be attributed to its redox/pH-responsive release properties of $\mathrm{CHX}$ and silver ions, leading to the synergistic efficiency of Ag-MSNs@CHX. In addition, the high-resolution images (Figure 7) also revealed that Ag-MSNs@CHX could adhere on the bacterial cell surface and induce bacterial cell death accompanied by decoherence of the extracellular matrix. Collectively, these findings demonstrated that Ag-MSNs@CHX efficiently inhibited $S$. mutans growth and its biofilm formation.

It is well known that high doses of silver ions or CHX can trigger oral cell death, resulting in unwanted side effects. ${ }^{48,49}$ We proposed that Ag-MSNs with a stimuli-responsive drug release behavior could reduce the toxicity of silver ions and CHX when exposed to normal cells. We, therefore, determined the viability of HIOECs after exposure to Ag-MSNs@CHX for 6 hours via the MTS assay (Figure 8). When HIOECs were treated with Ag-MSNs@CHX at the
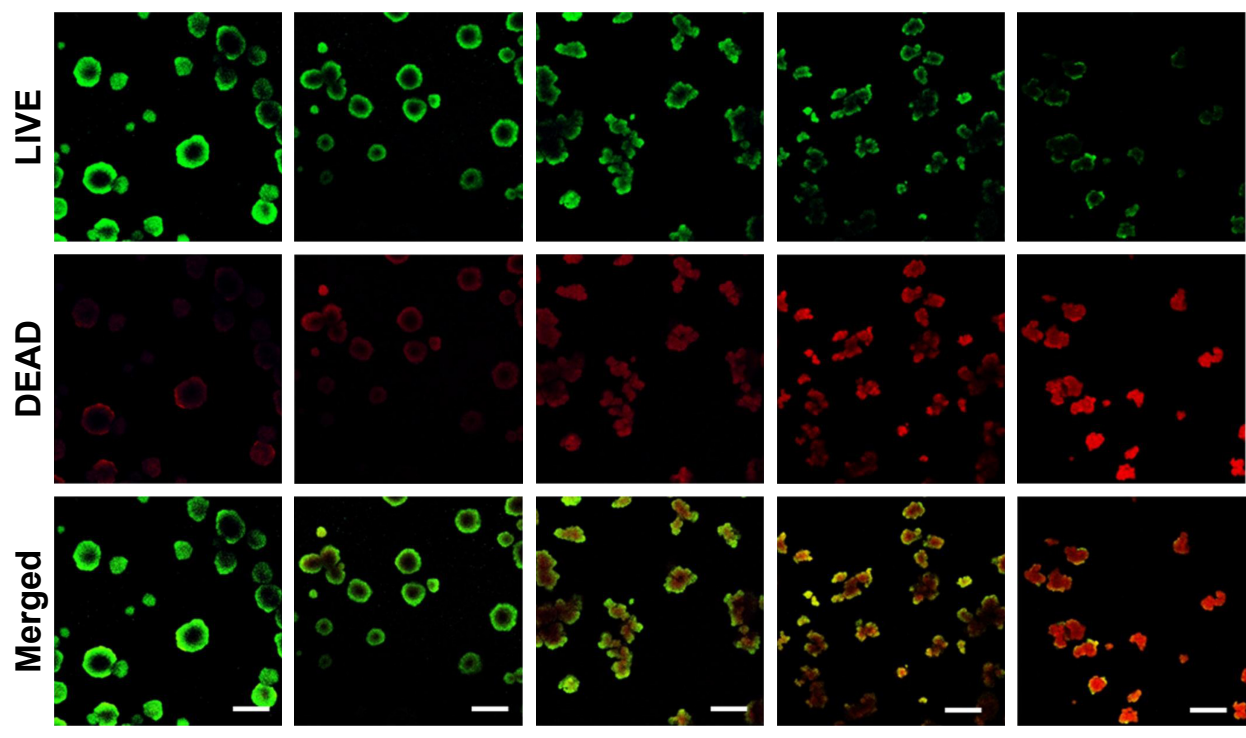

CON

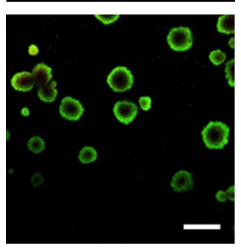

$\mathrm{AgNO}_{3}$
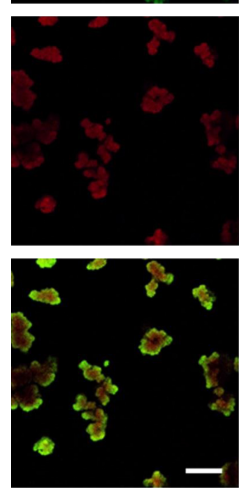

Ag-MSNs
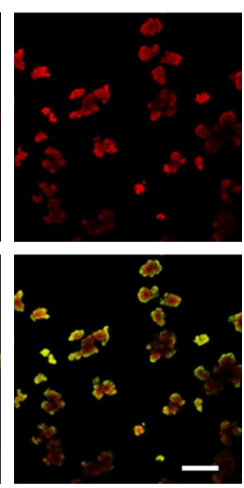

$\mathrm{CHX}$
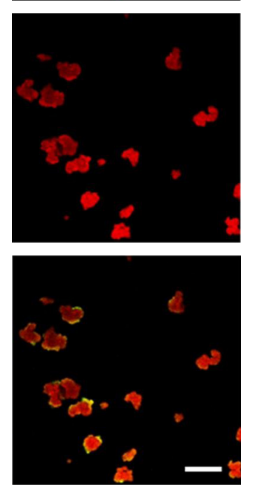

Ag-MSNs@

$\mathrm{CHX}$

Figure 5 Streptococcus mutans biofilms were formed in the presence of $\mathrm{Ag}-\mathrm{MSNs} @ \mathrm{CHX}$ or an equal amount of $\mathrm{Ag}-\mathrm{MSNs}$, free $\mathrm{CHX}$, or $\mathrm{AgNO}$.

Notes: The resulting biofilms were stained with the LIVE/DEAD kit after 24 hours and visualized by confocal laser scanning microscopy. The scale bars represent I00 $\mu \mathrm{m}$. Abbreviations: Ag-MSNs, silver-decorated mesoporous silica nanoparticles; Ag-MSNs@CHX, chlorhexidine-loaded, silver-decorated mesoporous silica nanoparticles; $\mathrm{CHX}$, chlorhexidine; CON, control. 

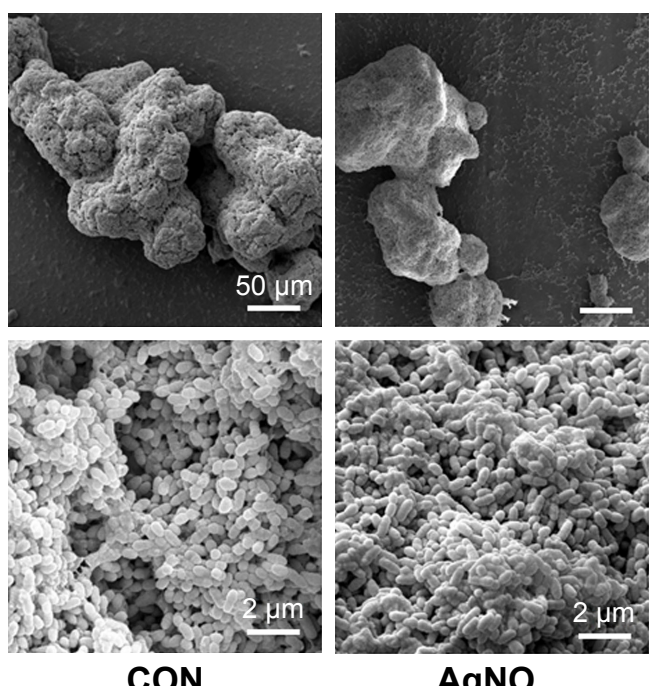

$\mathrm{AgNO}_{3}$
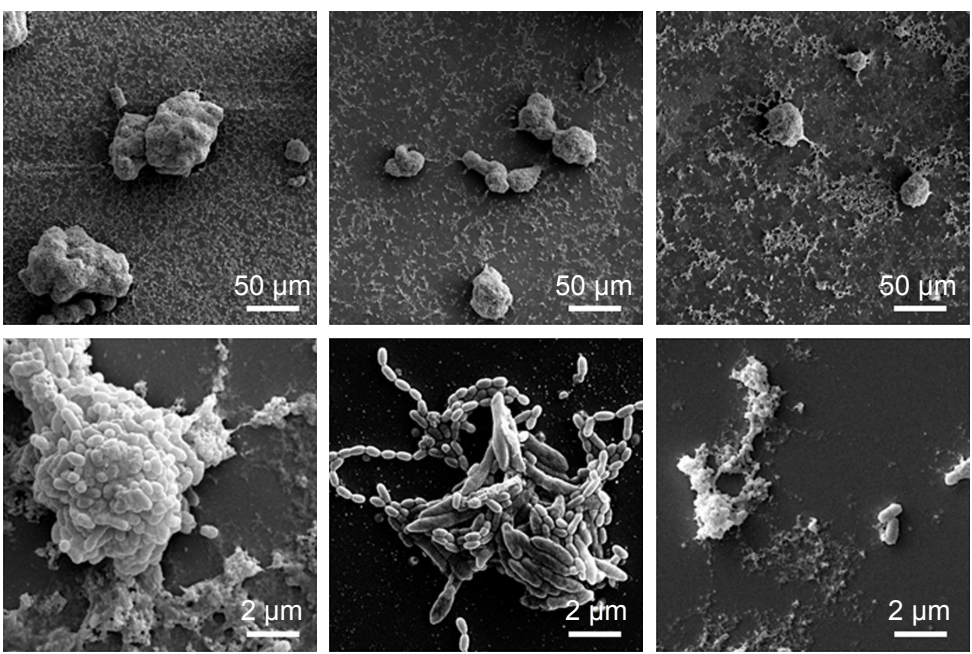

Ag-MSNs

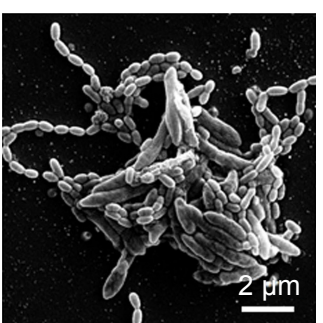

$\mathrm{CHX}$

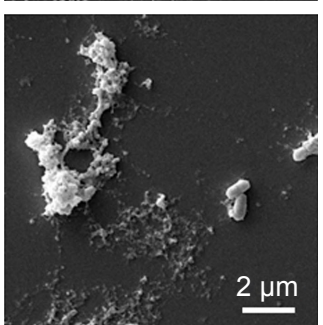

Ag-MSNs@CHX

Figure 6 SEM images of an Streptococcus mutans biofilm treated with Ag-MSNs@CHX or an equal amount of Ag-MSNs, free CHX, or AgNO for 24 hours.

Abbreviations: Ag-MSNs, silver-decorated mesoporous silica nanoparticles; Ag-MSNs@CHX, chlorhexidine-loaded, silver-decorated mesoporous silica nanoparticles; $\mathrm{CHX}$, chlorhexidine; CON, control; SEM, scanning electron microscopy.

MBIC $(50 \mu \mathrm{g} / \mathrm{mL})$, they retained $60 \%$ cell viability, indicating that Ag-MSNs@CHX, even at high concentrations, were well tolerated. In contrast, the CHX group exhibited significant cytotoxicity compared to the Ag-MSNs@CHX group, which was in line with the slow-release behavior of Ag-MSNs@CHX in the neutral microenvironment of normal cells. To further study the in vivo toxicity of this nanocarrier because of its possibility of oral exposure, we systematically evaluated the body weight, hematology, blood biochemistry, and organ histopathology of mice after

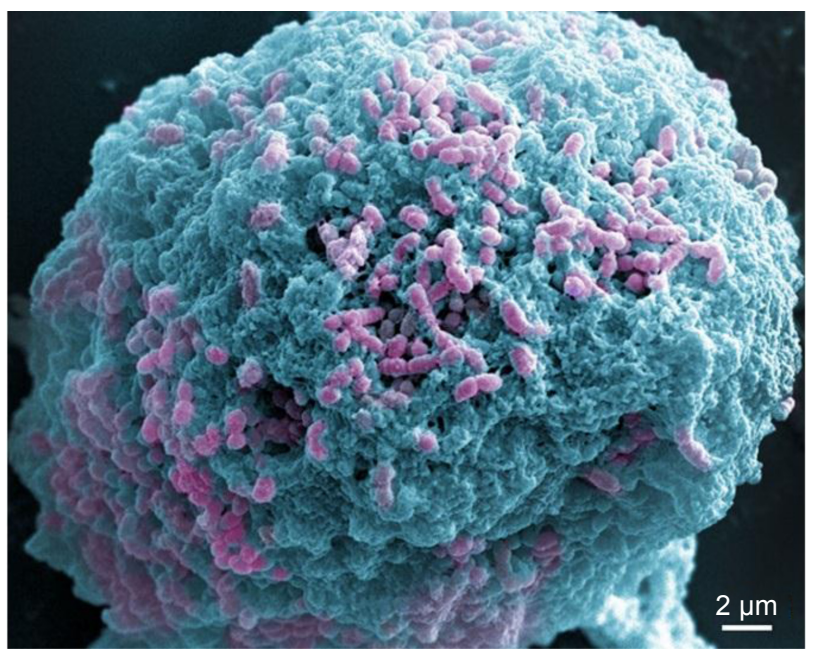

Figure 7 SEM image of an Streptococcus mutans biofilm treated with Ag-MSNs for 24 hours.

Note: The image is presented with pseudo-color to identify the bacteria (pink) and nanoparticle (cyan).

Abbreviations: Ag-MSNs, silver-decorated mesoporous silica nanoparticles; SEM, scanning electron microscopy. oral administration of $50 \mathrm{mg} / \mathrm{kg}$ of MSNs or Ag-MSNs. During the entire experimental period, neither death nor abnormal behavior was observed. Compared with the control group, no remarkable changes were found in the body weight of any of the groups (Figure 9). As shown in Figures 10 and 11, hematology parameters, including white blood cells, red blood cells, hematocrit, mean corpuscular volume, hemoglobin, platelets, mean corpuscular hemoglobin, and mean corpuscular hemoglobin concentration, as well as blood biochemistry parameters, including AST, ALT, ALP, ALB, blood urea nitrogen, serum creatinine, total cholesterol, and total triglycerides, were normal in the nanocarrier-treated groups compared with those of the control group, and they were within the normal ranges.

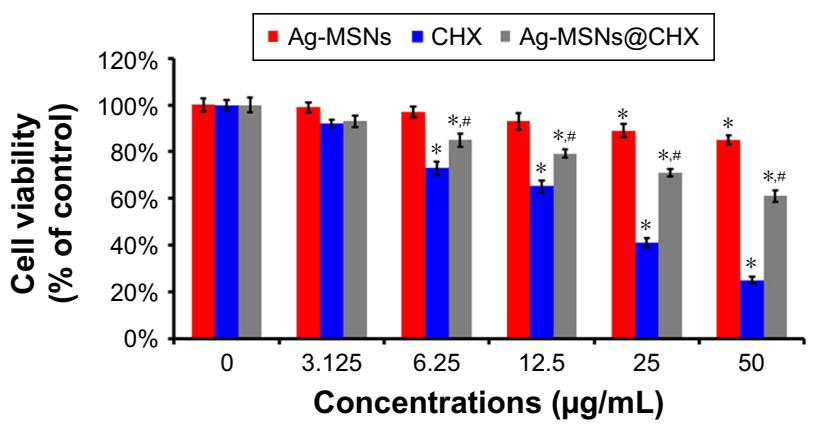

Figure 8 The cytotoxicity of Ag-MSNs@CHX, Ag-MSNs, and free $\mathrm{CHX}$ at different concentrations against human immortalized oral epithelial cells after 6 hours. Notes: These data represent three separate experiments and are presented as mean \pm SD. $* P<0.05$ vs the control group, ${ }^{*} P<0.05$ vs the $C H X$ group.

Abbreviations: Ag-MSNs, silver-decorated mesoporous silica nanoparticles; Ag-MSNs@CHX, chlorhexidine-loaded, silver-decorated mesoporous silica nanoparticles; $\mathrm{CHX}$, chlorhexidine. 


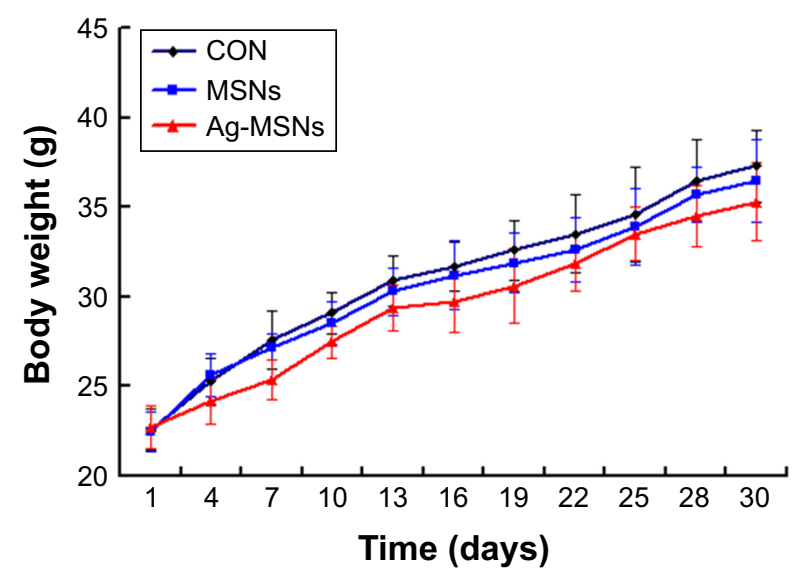

Figure 9 Body weights of mice from the control, MSN-treated, and Ag-MSNtreated groups 30 days after treatment.

Note: Each bar represents mean $\pm S D, n=6$.

Abbreviations: Ag-MSNs, silver-decorated mesoporous silica nanoparticles; CON, control; MSNs, mesoporous silica nanoparticles.

Furthermore, the H\&E staining assay also demonstrated a lack of evident pathological changes in the heart, liver, spleen, lung, and kidney in all groups (Figure 12). Taken together, these findings suggest that Ag-MSNs@CHX significantly reduced the toxicity of $\mathrm{CHX}$ in oral epithelial cells, and nanocarriers did not exhibit any abnormal effects on mice after oral administration.

\section{Conclusion}

We developed biodegradable disulfide-bridged MSNs to codeliver CHX and Ag NPs against oral pathogens. Benefitting from the GSH-triggered matrix degradation properties, AgMSNs@CHX with high loading contents showed sequential release of $\mathrm{CHX}$ and silver ions in response to both reducing and acidic microenvironments. Therefore, the proposed formulation significantly inhibited the growth of $S$. mutans and its biofilm. Importantly, Ag-MSNs@CHX were more effective than an equivalent amount of free CHX in limiting $S$. mutans biofilm formation by inducing bacterial cell death, particularly in the long term. Furthermore, Ag-MSNs markedly reduced the toxicity of $\mathrm{CHX}$ in oral epithelial cells and did not exhibit any abnormal effects on mice after oral exposure. Given the importance of inhibiting S. mutans and its biofilms efficiently and safely, the Ag-MSNs@CHX described here may have translational potential as a new type of nanoantiseptics for oral biofilm therapies.
A

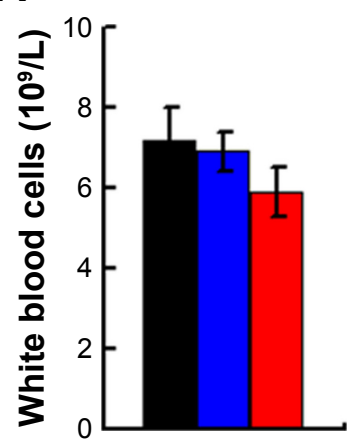

E

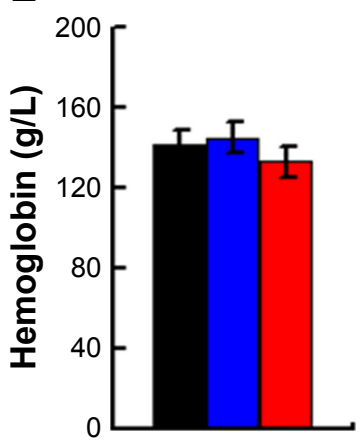

B

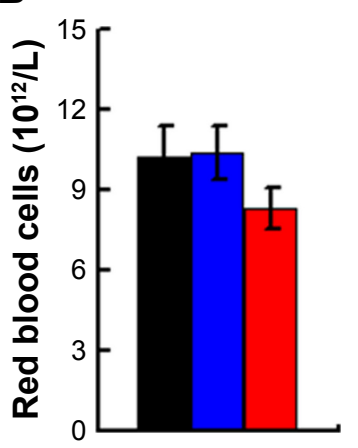

$\mathbf{F}$

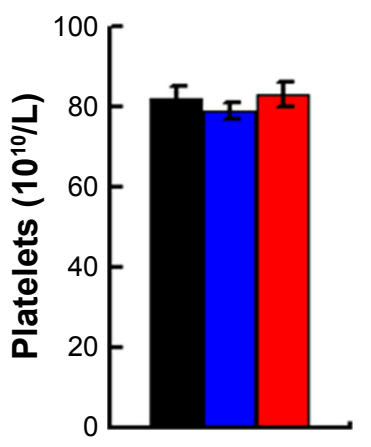

C

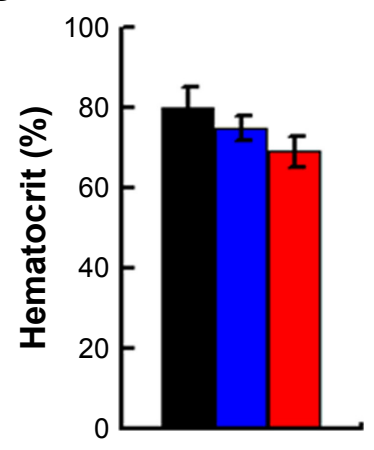

G

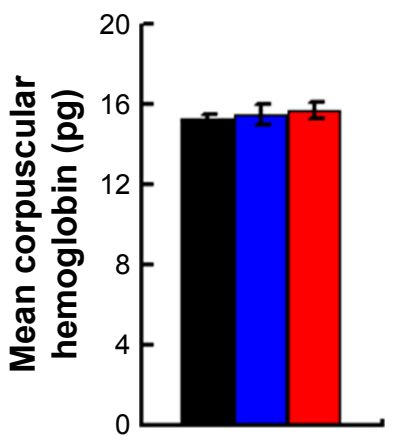

D

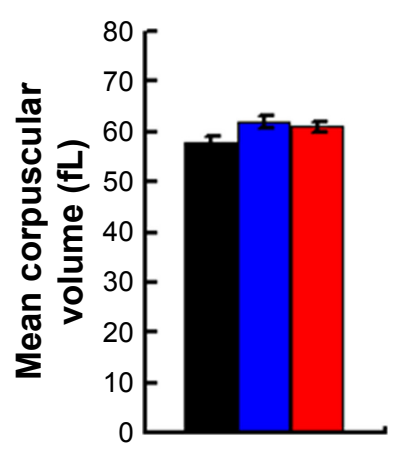

H

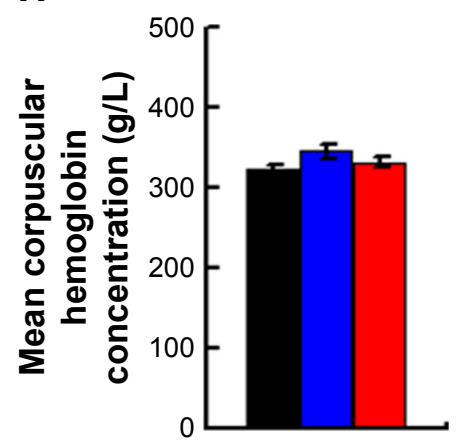

CON $\square$ MSNs $\square$ Ag-MSNs

Figure 10 Hematology data for the oral exposure of Ag-MSNs.

Notes: The changes in white blood cells $(\mathbf{A})$, red blood cells (B), hematocrit (C), mean corpuscular volume (D), hemoglobin (E), platelets (F), mean corpuscular hemoglobin (G), and mean corpuscular hemoglobin concentration $(\mathbf{H})$ in control mice, MSN-treated mice, and Ag-MSN-treated mice after 30 days of treatment. Each bar represents mean $\pm S D, n=6$.

Abbreviations: Ag-MSNs, silver-decorated mesoporous silica nanoparticles; CON, control; MSNs, mesoporous silica nanoparticles. 
A

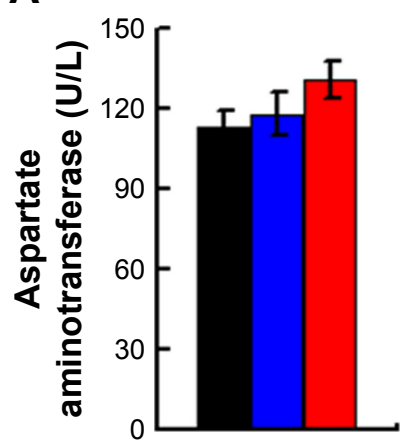

E

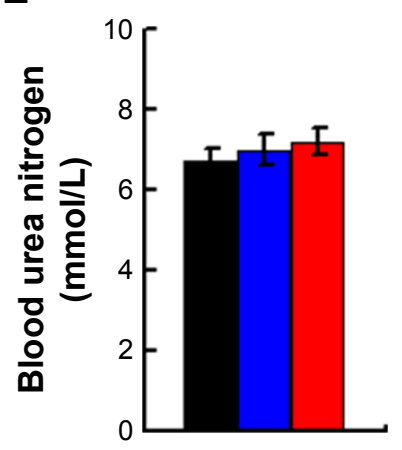

B

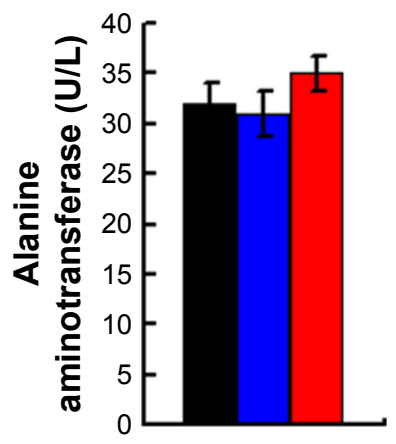

F

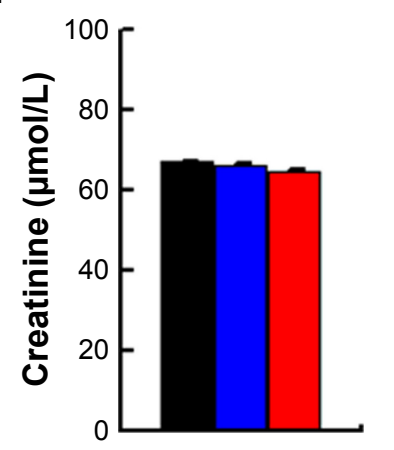

C

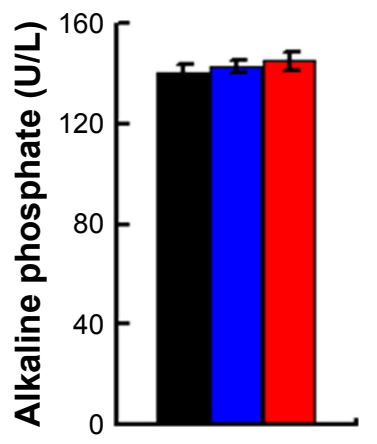

G

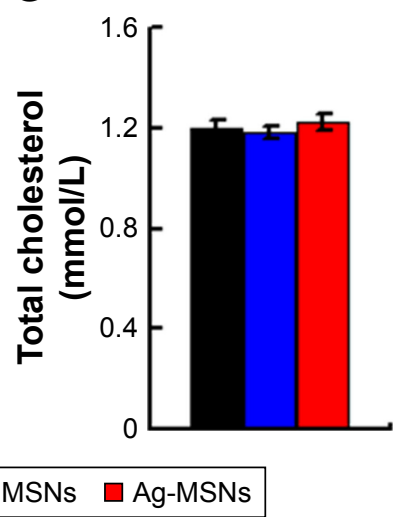

D

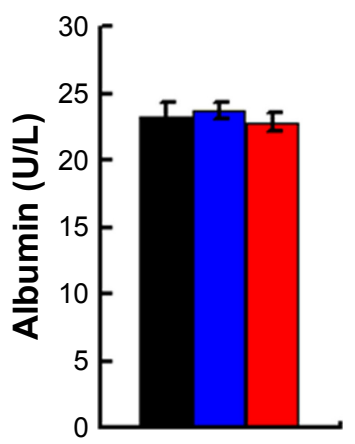

H

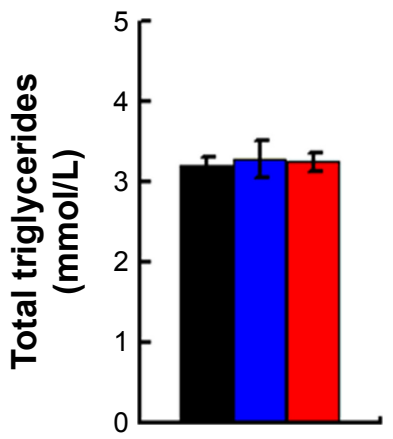

Figure I I Blood biochemistry data for the oral exposure of Ag-MSNs.

Notes: The changes in aminotransferase $(\mathbf{A})$, alanine aminotransferase (B), alkaline phosphatase (C), albumin (D), blood urea nitrogen (E), serum creatinine (F), total cholesterol $(\mathbf{G})$ and total triglycerides $(\mathbf{H})$ in control mice, MSN-treated mice, and Ag-MSN-treated mice after 30 days of treatment. Each bar represents mean \pm SD, $n=6$.

Abbreviations: Ag-MSNs, silver-decorated mesoporous silica nanoparticles; MSNs, mesoporous silica nanoparticles.
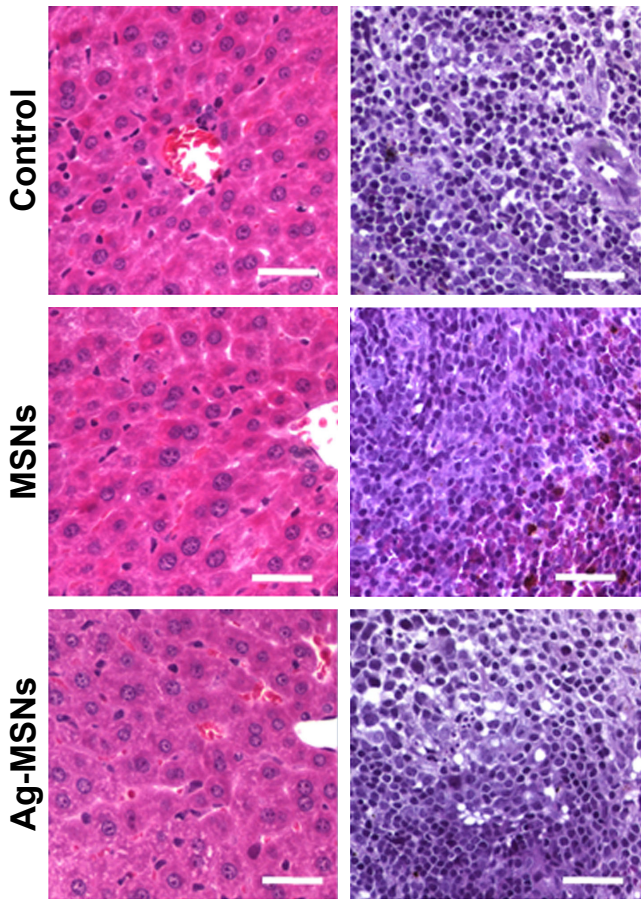

Liver

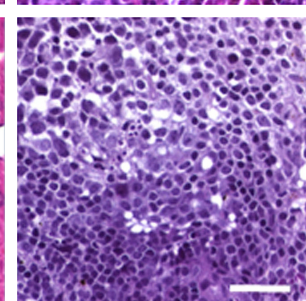

Spleen
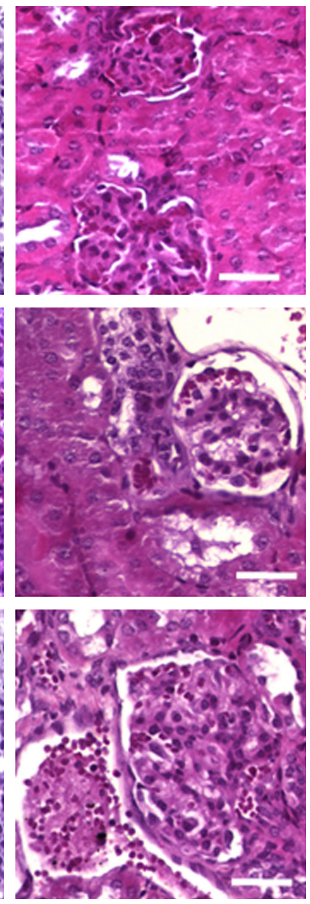

Kidney
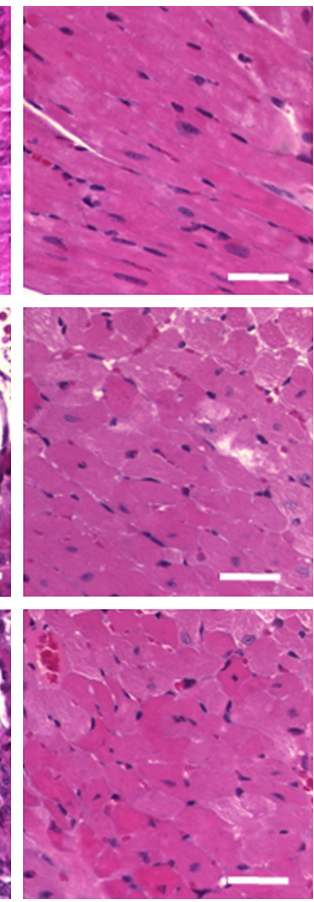

Heart
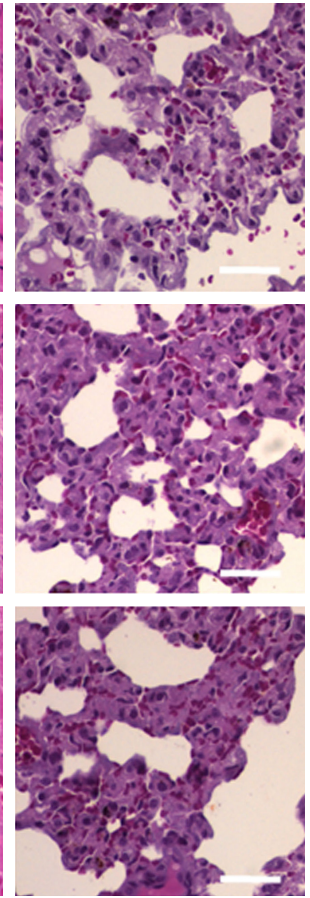

Lung

Figure 12 Representative H\&E-stained images of major organs, including the liver, spleen, kidneys, heart, and lungs, collected from control mice.

Notes: MSN-treated mice, and Ag-MSN-treated mice after 30 days of treatment. The scale bars represent $50 \mu \mathrm{m}$.

Abbreviations: Ag-MSNs, silver-decorated mesoporous silica nanoparticles; MSNs, mesoporous silica nanoparticles. 


\section{Acknowledgments}

This work was supported by the National Natural Science Foundation of China (grant numbers 81601609 and 81470778), the "Southeast University and Nanjing Medical University Cooperative Research Project" (2242018K3DN03), and the Science and Technology Commission Program of Nanjing (201605011).

\section{Disclosure}

The authors report no conflicts of interest in this work.

\section{References}

1. Kinane DF, Stathopoulou PG, Papapanou PN. Periodontal diseases. Nat Rev Dis Primers. 2017;3:17038.

2. Tonetti MS, Jepsen S, Jin L, Otomo-Corgel J. Impact of the global burden of periodontal diseases on health, nutrition and wellbeing of mankind: a call for global action. J Clin Periodontol. 2017;44(5):456-462.

3. Khan SA, Kong EF, Meiller TF, Jabra-Rizk MA. Periodontal diseases: bug induced, host promoted. PLoS Pathog. 2015;11(7):e1004952.

4. York A. Biofilms: the architect of the biofilm. Nat Rev Microbiol. 2017; 15(11):642-643.

5. Wu H, Moser C, Wang HZ, Høiby N, Song ZJ. Strategies for combating bacterial biofilm infections. Int J Oral Sci. 2015;7(1):1-7.

6. Ferrer MD, Mira A. Oral biofilm architecture at the microbial scale. Trends Microbiol. 2016;24(4):246-248.

7. Vieira Colombo AP, Magalhães CB, Hartenbach FA, Martins do Souto R, Maciel da Silva-Boghossian C. Periodontal-disease-associated biofilm: a reservoir for pathogens of medical importance. Microb Pathog. 2016;94:27-34.

8. Klein MI, Hwang G, Santos PH, Campanella OH, Koo H. Streptococcus mutans-derived extracellular matrix in cariogenic oral biofilms. Front Cell Infect Microbiol. 2015;5:10.

9. Kreth J, Zhang Y, Herzberg MC. Streptococcal antagonism in oral biofilms: Streptococcus sanguinis and Streptococcus gordonii interference with Streptococcus mutans. J Bacteriol. 2008;190(13):4632-4640.

10. Xiao J, Hara AT, Kim D, Zero DT, Koo H, Hwang G. Biofilm threedimensional architecture influences in situ $\mathrm{pH}$ distribution pattern on the human enamel surface. Int J Oral Sci. 2017;9(2):74-79.

11. Lebeaux D, Ghigo JM, Beloin C. Biofilm-related infections: bridging the gap between clinical management and fundamental aspects of recalcitrance toward antibiotics. Microbiol Mol Biol Rev. 2014;78(3): 510-543.

12. Mah TF, O'Toole GA. Mechanisms of biofilm resistance to antimicrobial agents. Trends Microbiol. 2001;9(1):34-39.

13. Percival SL, McCarty SM. Silver and alginates: role in wound healing and biofilm control. Adv Wound Care (New Rochelle). 2015;4(7):407-414.

14. Abdulkareem EH, Memarzadeh K, Allaker RP, Huang J, Pratten J, Spratt D. Anti-biofilm activity of zinc oxide and hydroxyapatite nanoparticles as dental implant coating materials. J Dent. 2015;43(12): 1462-1469.

15. Jones CG. Chlorhexidine: is it still the gold standard? Periodontol 2000. 1997; 15(1):55-62.

16. Hope CK, Wilson M. Analysis of the effects of chlorhexidine on oral biofilm vitality and structure based on viability profiling and an indicator of membrane integrity. Antimicrob Agents Chemother. 2004;48(5): 1461-1468.

17. Shen Y, Stojicic S, Haapasalo M. Antimicrobial efficacy of chlorhexidine against bacteria in biofilms at different stages of development. J Endod. 2011;37(5):657-661.

18. Rizzello L, Pompa PP. Nanosilver-based antibacterial drugs and devices: mechanisms, methodological drawbacks, and guidelines. Chem Soc Rev. 2014;43(5):1501-1518.
19. Franci G, Falanga A, Galdiero S, et al. Silver nanoparticles as potential antibacterial agents. Molecules. 2015;20(5):8856-8874.

20. Singh P, Pandit S, Garnæs J, et al. Green synthesis of gold and silver nanoparticles from Cannabis sativa (industrial hemp) and their capacity for biofilm inhibition. Int J Nanomedicine. 2018;13:3571-3591.

21. Monteiro DR, Silva S, Negri M, et al. Antifungal activity of silver nanoparticles in combination with nystatin and chlorhexidine digluconate against Candida albicans and Candida glabrata biofilms. Mycoses. 2013;56(6):672-680.

22. Ben-Knaz R, Pedahzur R, Avnir D. Bioactive doped metals: high synergism in the bactericidal activity of chlorhexidine@silver towards wound pathogenic bacteria. RSC Adv. 2013;3(21):8009-8015.

23. Supranoto SC, Slot DE, Addy M, Van der Weijden GA. The effect of chlorhexidine dentifrice or gel versus chlorhexidine mouthwash on plaque, gingivitis, bleeding and tooth discoloration: a systematic review. Int J Dent Hyg. 2015;13(2):83-92.

24. Song Y, Li Y, Xu Q, Liu Z. Mesoporous silica nanoparticles for stimuliresponsive controlled drug delivery: advances, challenges, and outlook. Int J Nanomedicine. 2017;12:87-110.

25. Kang T, Li F, Baik S, Shao W, Ling D, Hyeon T. Surface design of magnetic nanoparticles for stimuli-responsive cancer imaging and therapy. Biomaterials. 2017;136:98-114.

26. Yang K, Feng L, Liu Z. Stimuli responsive drug delivery systems based on nano-graphene for cancer therapy. Adv Drug Deliv Rev. 2016; 105(Pt B):228-241.

27. Zheng $\mathrm{X}$, Zhang F, Zhao Y, et al. Self-assembled dual fluorescence nanoparticles for CD44-targeted delivery of anti-miR-27a in liver cancer theranostics. Theranostics. 2018;8(14):3808-3823.

28. Bharti C, Nagaich U, Pal AK, Gulati N. Mesoporous silica nanoparticles in target drug delivery system: a review. Int J Pharm Investig. 2015; 5(3):124-133.

29. Shao D, Li J, Zheng X, et al. Janus "nano-bullets" for magnetic targeting liver cancer chemotherapy. Biomaterials. 2016;100:118-133.

30. Shao D, Lu MM, Zhao YW, et al. The shape effect of magnetic mesoporous silica nanoparticles on endocytosis, biocompatibility and biodistribution. Acta Biomater. 2017;49:531-540.

31. Wang Z, Chang Z, Lu M, et al. Janus silver/silica nanoplatforms for light-activated liver cancer chemo/photothermal therapy. ACS Appl Mater Interfaces. 2017;9(36):30306-30317.

32. Wang Z, Shao D, Chang Z, et al. Janus gold nanoplatform for synergetic chemoradiotherapy and computed tomography imaging of hepatocellular carcinoma. ACS Nano. 2017;11(12):12732-12741.

33. Wang Z, Chang Z, Lu M, et al. Shape-controlled magnetic mesoporous silica nanoparticles for magnetically-mediated suicide gene therapy of hepatocellular carcinoma. Biomaterials. 2018;154:147-157.

34. Chang ZM, Wang Z, Shao D, et al. Shape engineering boosts magnetic mesoporous silica nanoparticle-based isolation and detection of circulating tumor cells. ACS Appl Mater Interfaces. 2018;10(13): 10656-10663.

35. Wang Y-S, Shao D, Zhang L, et al. Gold nanorods-silica Janus nanoparticles for theranostics. Appl Phys Lett. 2015;106(17):173705.

36. Kuthati Y, Kankala RK, Lin SX, Weng CF, Lee CH. pH-triggered controllable release of silver-indole-3 acetic acid complexes from mesoporous silica nanoparticles (IBN-4) for effectively killing malignant bacteria. Mol Pharm. 2015;12(7):2289-2304.

37. Chang ZM, Wang Z, Lu MM, et al. Janus silver mesoporous silica nanobullets with synergistic antibacterial functions. Colloids Surf B Biointerfaces. 2017;157:199-206.

38. Shao D, Wang Z, Dong WF, et al. Facile synthesis of core-shell magnetic mesoporous silica nanoparticles for $\mathrm{pH}$-sensitive anticancer drug delivery. Chem Biol Drug Des. 2015;86(6):1548-1553.

39. Chang Z-M, Wang Z, Shao D, et al. Fluorescent-magnetic Janus nanorods for selective capture and rapid identification of foodborne bacteria. Sens Actuators B Chem. 2018;260:1004-1011.

40. Şen Karaman D, Manner S, Rosenholm JM. Mesoporous silica nanoparticles as diagnostic and therapeutic tools: how can they combat bacterial infection? Ther Deliv. 2018;9(4):241-244. 
41. Croissant JG, Fatieiev Y, Khashab NM. Degradability and clearance of silicon, organosilica, silsesquioxane, silica mixed oxide, and mesoporous silica nanoparticles. Adv Mater. Epub 13 Jan 2017.

42. Yue J, Luo SZ, Lu MM, Shao D, Wang Z, Dong WF. A comparison of mesoporous silica nanoparticles and mesoporous organosilica nanoparticles as drug vehicles for cancer therapy. Chem Biol Drug Des. 2018; 92(2):1435-1444.

43. Shao D, Li M, Wang Z, et al. Bioinspired diselenide-bridged mesoporous silica nanoparticles for dual-responsive protein delivery. $A d v$ Mater. 2018;30:1801198.

44. du X, Li X, Xiong L, Zhang X, Kleitz F, Qiao SZ. Mesoporous silica nanoparticles with organo-bridged silsesquioxane framework as innovative platforms for bioimaging and therapeutic agent delivery. Biomaterials. 2016;91:90-127.

45. du X, Kleitz F, Li X, Huang H, Zhang X, Qiao SZ. Disulfide-bridged organosilica frameworks: designed, synthesis, redox-triggered biodegradation, and nanobiomedical applications. Adv Funct Mater. Epub 25 Apr 2018.
46. Lu MM, Wang QJ, Chang ZM, et al. Synergistic bactericidal activity of chlorhexidine-loaded, silver-decorated mesoporous silica nanoparticles. Int J Nanomedicine. 2017;12:3577-3589.

47. Zhong LP, Pan HY, Zhou XJ, et al. Characteristics of a cancerous cell line, HIOEC-B(a)P-96, induced by benzo(a)pyrene from human immortalized oral epithelial cell line. Arch Oral Biol. 2008;53(5):443-452.

48. Müller HD, Eick S, Moritz A, Lussi A, Gruber R. Cytotoxicity and antimicrobial activity of oral rinses in vitro. Biomed Res Int. 2017;2017: 4019723.

49. Gaillet S, Rouanet JM. Silver nanoparticles: their potential toxic effects after oral exposure and underlying mechanisms - a review. Food Chem Toxicol. 2015;77:58-63.
International Journal of Nanomedicine

\section{Publish your work in this journal}

The International Journal of Nanomedicine is an international, peerreviewed journal focusing on the application of nanotechnology in diagnostics, therapeutics, and drug delivery systems throughout the biomedical field. This journal is indexed on PubMed Central,

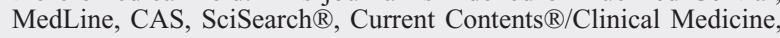

\section{Dovepress}

Journal Citation Reports/Science Edition, EMBase, Scopus and the Elsevier Bibliographic databases. The manuscript management system is completely online and includes a very quick and fair peer-review system, which is all easy to use. Visit http://www.dovepress.com/ testimonials.php to read real quotes from published authors. 